\title{
SMOOTH HOMOGENEOUS ASYMPTOTICALLY STABILIZING FEEDBACK CONTROLS
}

\author{
HENRY HERMES
}

\begin{abstract}
If a smooth nonlinear affine control system has a controllable linear approximation, a standard technique for constructing a smooth (linear) asymptotically stabilizing feedback control is via the LQR (linear, quadratic, regulator) method. The nonlinear system may not have a controllable linear approximation, but instead may be shown to be small (or large) time locally controllable via a high order, homogeneous approximation. In this case one can attempt to construct an asymptotically stabilizing feedback control as the optimal control, relative to a cost functional with homogeneous integrand, for the approximating system. Necessary, and some sufficient, conditions for the existence of a smooth (real analytic), stabilizing feedback control of this form are given. For some systems which satisfy these necessary conditions, the specific form of a stabilizing control is established.
\end{abstract}

\section{INTRODUCTION}

This paper deals with the problem of existence and construction of a smooth, asymptotically stabilizing (state) feedback control (hereafter ASFC) for a real analytic, $n \geq 2$ dimensional, control system of the form

$$
\dot{x}=X(x)+u Y(x), \quad X(0)=0, \quad Y(0) \neq 0 .
$$

If one can find a smooth, homogeneous (of appropriate order) ASFC for a homogeneous approximating system of (1.1), then (see [10], [19]) this control is also a local ASFC for (1.1). Our goal is to characterize all homogeneous (approximating) systems which admit a smooth $\left(C^{\infty}\right)$, homogeneous ASFC as an optimal control of a nonlinear regulator problem.

For example, suppose the linearization of (1.1) is a controllable linear system $\dot{x}=A x+u b$. Assign the quadratic cost functional $C(u)=\int_{0}^{\infty}\left[e u^{2}(t)+\right.$ $\left.h_{2}^{+}(x(t))\right] d t$ where $h_{2}^{+}$is a positive definite quadratic form (homogeneous of degree 2 with respect to the standard dilation $\left.\delta_{\varepsilon}^{1} x=\left(\varepsilon x_{1}, \ldots, \varepsilon x_{n}\right)\right)$. Classical theory, [17], shows that the value function $v$ is a smooth, positive definite, solution of a smooth Hamilton-Jacobi-Bellman (hereafter HJB) equation. If one considers $b$ as a vector field $Y$ on $\mathbb{R}^{n}$, i.e., let $Y=\sum b_{i} \frac{\partial}{\partial x_{i}}$, then the linear optimal control is an ASFC and has the form $u^{*}(x)=-\gamma(Y v)(x)$,

Department of Mathematics, Box 395, University of Colorado, Boulder, CO $80309-$ 0395, USA.

Received by the journal January 25, 1996. Accepted for publication December 19, 1996.

This research was funded by NSF grant DMS 9530973.

(c) Société de Mathématiques Appliquées et Industrielles. Typeset by $\mathcal{A} \mathcal{M} \mathcal{S}_{\text {-TEX }}$ 
where $\gamma>0$ is a gain constant. Furthermore, a controllable linear system can always be transformed to "first power integrator form," i.e. $\dot{y}_{1}=y_{2}$, $\dot{y}_{2}=y_{3}, \ldots, \dot{y}_{n}=u$ via linear feedback and a linear coordinate change. For nonlinear systems of the form (1.1) which have controllable linearizations (homogeneous approximations relative to $\delta_{\varepsilon}^{1}$ ), the first power integrator can be considered a "nice" canonical representative of these approximations under the linear feedback group. An ASFC for the integrator easily transforms to an ASFC of any controllable, linear system, i.e. system in the orbit of the group acting on the integrator.

First power integrators are a special case of the $p$ th power integrators

$$
\dot{y}_{1}=y_{2}^{p}, \quad \dot{y}_{2}=y_{3}^{p}, \ldots, \dot{y}_{n-1}=y_{n}^{p}, \quad \dot{y}_{n}=u
$$

with $p$ an odd integer. In [8], an inductive argument is used to show the odd $p$ th power integrator admits an ASFC of the form $u^{*}(y)=-\gamma\left(\sum_{1}^{n} \alpha_{i} y_{i}\right)^{p}$ and quadratic Lyapunov function $v(y)=(1 / 2) y^{T} Q y$. We will show that $v$ is a value function for a cost functional of the form $C(u)=\int_{0}^{\infty}\left[e u^{p+1}(t)+\right.$ $\left.h_{p+1}^{+}(y(t))\right] d t$ where $h_{p+1}^{+}$is homogeneous of degree $(p+1)$ with respect to the standard dilation, positive definite, and smooth. Letting $X(y)=$ $\sum_{j=1}^{n-1} y_{j+1}^{p} \frac{\partial}{\partial y_{j}}, Y=\frac{\partial}{\partial y_{n}}$ the value function $v$ is smooth, homogeneous of degree 2 with respect to $\delta_{\varepsilon}^{1}$, and is a positive definite smooth solution of the HJB equation $-\gamma((Y v)(y))^{p+1}+(X v)(y)=-h_{p+1}^{+}(y)$. Furthermore, the smooth, homogeneous ASFC $u^{*}$ has the form $u^{*}(y)=-\gamma((Y v)(y))^{p}$.

As in the linear system case, i.e., $p=1$, the odd, $p$ th power integrator system (1.2) should be considered a canonical representative for its "feedback equivalence class" where here feedback and coordinate changes should be such as to preserve the degree of homogeneity of the vector fields $X, Y$ which describe the system. For a more general dilation $\delta_{\varepsilon}^{r}$ on $\mathbb{R}^{n}$, this leads to the notion of the " $\delta_{\varepsilon}^{r}$ feedback equivalence group." Again, as in the linear case, once a smooth, homogeneous ASFC is known for a canonical representative, it is easily transformed to any system in the equivalence class. Thus the goal is to characterize the $\delta_{\varepsilon}^{r}$ feedback group equivalence classes of homogeneous systems which admit a smooth, homogeneous, ASFC as an optimal control of a nonlinear regulator problem, and to find a (nice) canonical representative system from each such equivalence class. The $p$ th power integrators, $p=1,3, \ldots$, would be considered as nice canonical representatives from their equivalence classes.

Our method is to first assign weights, wt $X=k$, wt $Y \geq 1$ to the "symbols" $X, Y$, of the vector fields $X, Y$ of (1.1). The weight of a Lie product is the sum of the weights of its factors. We then consider the free Lie Algebra on these symbols, and a homomorphism from this free Lie algebra to the Lie algebra generated by the vector fields $X, Y$, see [22] for details. We will, however, abuse notation, and use $L(X, Y)$ as either the free Lie algebra on the symbols $X, Y$, or the Lie algebra generated by considering these as vector fields. In particular, we identify a given bracket in the free Lie algebra with the vector field resulting from this bracket of the vector fields $X, Y$. Clearly many different brackets in the free Lie algebra can map to the same vector field, but this presents no problem. The weight induced filtration of 
the Lie algebra, $L(X, Y)$, generated by $X, Y$, is used to induce (natural) local coordinates and a dilation, $\delta_{\varepsilon}^{r}, 1 \leq r_{1} \leq \cdots \leq r_{n}$, relative to which there is a homogeneous approximation (see eqs. (2.3), (2.4)) of system (1.1). A homogeneous ASFC of the approximating system, of appropriate order, is also a local ASFC of (1.1). We seek necessary conditions that such an ASFC exist as a smooth optimal control for a nonlinear regulator problem having positive definite, homogeneous, integrand.

If the vector field $X$, in (1.1), admits a homogeneous approximation $X^{(1-k)}$ such that the origin of $\dot{x}=X^{(1-k)}(x)$ is asymptotically stable, then ([10], [19]) the origin is locally asymptotically stable for $\dot{x}=X(x)$ and no control is necessary. Our interest is in the case when control is necessary to cause the solution from any initial data to tend to zero as $t \rightarrow \infty$. We define: an integral manifold $M^{k}$ of a vector field $Z, Z(0)=0$, is a stable manifold of the rest solution zero if there is a $C^{1}$, positive definite, function $v: \mathbb{R}^{n} \rightarrow \mathbb{R}^{1}$ such that $(Z v)(x)$ is negative definite on $M^{k}$. If $M^{k}$ is a stable manifold for the rest solution zero of $Z$, the solution $x(t, p)$ of $\dot{x}=Z(x)$, $x(0)=p$ will satisfy $x(t, p) \rightarrow 0$ as $t \rightarrow \infty$. We assume, throughout, that no such stable manifold of positive dimension $k$ exist for either $X$ or $X^{(1-k)}$. This assures that any solution of the controlled system which tends to zero as $t \rightarrow \infty$ does so because of control.

The main results, theorem 3.1 and theorem 3.8, can roughly be stated as follows. A necessary condition for the existence of a smooth ASFC which is an optimal control for a nonlinear regulator problem is that weight assignments can be made so that there are $n$ products of $X, Y$, having weight one, which are linearly independent when evaluated at zero, while products of weight less than or equal to zero vanish at zero. This means it is necessary that the induced dilation be $\delta_{\varepsilon}^{r}$ with $r=(1, \ldots, 1)$, which we call the standard dilation $\delta_{\varepsilon}^{1}$. It is also necessary that $k \in\{0,-2,-4, \ldots\}$. If $k=0$, the homogeneous approximation is a standard linear approximation, which gives the classical "linear, quadratic, regulator." If $k=(-p+1), p \geq 3$ and odd, one gets the $p$ th power integrators, but also other systems not in their $\delta_{\varepsilon}^{1}$ feedback equivalence class, which do admit a smooth, homogeneous, ASFC as an optimal control of a nonlinear regulator problem.

Brockett's necessary conditions, [4], for the existence of a continuous ASFC, which is only a function of the state, showed that STLC at zero was not sufficient. In dimension $n \geq 4$, Coron, [6], shows that STLC does imply the existence of a continuous (time periodic) "dynamic" feedback control $u(t, x)$ which actually drives the system to zero in finite time. Other results along this line can be found in [16]. It is also true that systems of the form (1.1) can have a continuous, state dependent, ASFC, but no $C^{1}$ such control. For results on the differentiability properties of ASFC, in two dimensional systems, see [7].

\section{THE PRECISE PROBLEM FORMULATION}

Let $\delta_{\varepsilon}^{r}: \mathbb{R}^{n} \rightarrow \mathbb{R}^{n}$ be defined by $\delta_{\varepsilon}^{r} x=\left(\varepsilon^{r_{1}} x_{1}, \ldots, \varepsilon^{r_{n}} x_{n}\right)$ with $\varepsilon>0$ and $1 \leq r_{1} \leq \cdots \leq r_{n}$ integers. A smooth $\left(C^{\infty}\right)$ function $f: \mathbb{R}^{n} \rightarrow \mathbb{R}^{1}$ is homogeneous of degree $m$ with respect to $\delta_{\varepsilon}^{r}$, denoted $f \in P_{m}$, if $f\left(\delta_{\varepsilon}^{r} x\right)=$ 
$\varepsilon^{m} f(x)$. We set $P_{m}=\{0\}$ if $m<0$. A smooth vector field $X(x)=$ $\sum_{j=1}^{n} a_{j}(x) \frac{\partial}{\partial x_{j}}$ is homogeneous of degree $m$ with respect to $\delta_{\varepsilon}^{r}$ (in the given local coordinates, i.e., this is not a coordinate free notion) if $a_{j} \in P_{r_{j}+m-1}$, $j=1, \ldots, n$. In this case we write $X^{(m)}$ to denote homogeneity of degree $m$. It is worth remarking that the above definition of homogeneity of a function $f: \mathbb{R}^{n} \rightarrow \mathbb{R}^{1}$ is standard in the literature, but several definitions of homogeneity of vector fields can be found. In their classical paper [18], Rothschild and Stein define a vector field $X$ to be homogeneous of degree $m$ with respect to a dilation $\delta_{\varepsilon}^{r}$ if $X h \in P_{k-m}$ whenever $h \in P_{k}, k=0,1, \ldots$. With $X$ given in local coordinates, as above, this requires $a_{j} \in P_{r_{j}-m}$ and a vector field which is linear in the local coordinates becomes homogeneous of degree zero with respect to the standard dilation $\delta_{\varepsilon}^{1}$, while a linear map from $\mathbb{R}^{n}$ to $\mathbb{R}^{n}$ is homogeneous of degree one. The definition of vector field homogeneity given here makes either a map or vector field, which is linear in the local coordinates, homogeneous of degree one with respect to the standard dilation. For an excellent discussion of advantages and disadvantages of the various definitions, see [15].

Assume, without loss of generality, that feedback has been used, and local coordinates chosen, in (1.1) so that

$$
X(x)=\sum_{j=2}^{n} a_{j}(x) \frac{\partial}{\partial x_{j}}, \quad Y(x)=\frac{\partial}{\partial x_{1}} .
$$

Let $\alpha>0, \Omega^{\alpha}$ denote the set of Lebesgue measurable (open loop) controls $u:[0, \infty) \rightarrow[-\alpha, \alpha]$ and $A_{1}^{\alpha}(t)$ be the set of all points which can be reached at time $t$ by solutions of (1.1), from initial data $x(0)=0$, and with controls $u \in \Omega^{\alpha}$. As is standard, $A_{1}^{\alpha}(t)$ will be referred to as the attainable set of system (1.1) at time $t \geq 0$. System (1.1) is small time locally controllable (STLC) at zero if for any $t_{1}>0, \alpha>0,0 \in \operatorname{int} A_{1}^{\alpha}\left(t_{1}\right)$. System (1.1) is large time locally controllable (LTLC) at zero if given any $\alpha>0$, there exists a $t_{1}>0$ such that $0 \in \operatorname{int} A_{1}^{\alpha}\left(t_{1}\right)$. STLC has been extensively studied, see [3], [21], with a major, computable, sufficient condition given by Sussmann in [22], and paraphrased as theorem 2.4 in what follows. A computable sufficient condition for LTLC is given in [11], and rephrased as theorem 2.6, below. Clearly STLC implies LTLC while (for an analytic system (1.1)) LTLC is sufficient for the Brockett necessary condition, [4], for the existence of an ASFC, i.e., from any initial point $y$ in a neighborhood of zero there exists an open loop control $u_{y} \in \Omega^{\alpha}$, with solution of (1.1) denoted $t \rightarrow$ $x\left(t, u_{y}\right)$, such that $\lim _{t \rightarrow \infty} x\left(t, u_{y}\right)=0$.

Given any dilation $\delta_{\varepsilon}^{r}$ and any local coordinates $x$, one can expand the vector fields $X, Y$ as

$$
\begin{aligned}
& X(x)=X^{(1-k)}(x)+X^{(2-k)}(x)+\cdots \\
& Y(x)=Y^{(1-m)}(x)+Y^{(2-m)}(x)+\cdots
\end{aligned}
$$

where we assume $X^{(1-k)}, Y^{(1-m)}$ are the lowest order nonvanishing terms. Then $X(0)=0$ implies $X^{(1-k)}(0)=0$ and $Y(0) \neq 0$ implies $m \geq 1$ and 
$Y^{(1-m)}(0) \neq 0$. The homogeneous approximation of system (1.1), relative to dilation $\delta_{\varepsilon}^{r}$ and given local coordinates, is

$$
\dot{x}=X^{(1-k)}(x)+u Y^{(1-m)}(x)
$$

Since we assume that the rest solution zero of the uncontrolled system (1.1) has no stable manifold of positive dimension, asymptotic stability can only result from use of control. To assure the Brockett necessary condition, we require that system (1.1) be either STLC (implies LTLC) or LTLC at zero. The following theorem relates STLC of the homogeneous approximation (2.1) to STLC or LTLC of system (1.1).

ThEOREM 2.1. ([3], [11]) If there exists a dilation $\delta_{\varepsilon}^{r}$ for which $k=0$ in the expansion of $X$, or $k \geq 0$ and $(m-k) \geq 0$, and the approximation system (1.2) is STLC at zero, then system (1.1) is STLC at zero. If $k \leq-1$ and system (1.2) is STLC at zero, system (1.1) is LTLC at zero.

The use of homogeneous approximations to construct an ASFC for (1.1) stems from the following theorem, which is stated, below, for smooth controls but holds under far weaker smoothness assumptions, see [19].

Theorem 2.2. ([10]) If $u^{*} \in P_{m-k}$ is an ASFC for the homogeneous approximating system (2.1), it is a local ASFC for system (1.1).

In light of the above two theorems, our goal will be to determine when an STLC homogeneous approximating system has a smooth, homogeneous (of appropriate degree) ASFC.

For applications and a reasonable theory, it is essential to have a constructive way of obtaining an appropriate dilation and local coordinates, and these should come in a coordinate free way from system (1.1). Let $[X, Y]$ denote the Lie product of the vector fields $X, Y, L(X, Y)$ the Lie algebra generated by $X, Y$ under this product and $L(X, Y)(0)$ the elements of $L(X, Y)$ evaluated at zero. We assume, throughout, that $\operatorname{dim} L(X, Y)(0)=n$. An extended filtration, $\mathcal{F}$, of $L(X, Y)$ at zero is a sequence of subspaces $\left\{F_{j}:-\infty<j<\infty\right\}$ of $L(X, Y)$ such that for all integers $i, j$ :

$$
\begin{array}{ll}
\text { (i) } \quad F_{j} \subset F_{j+1} & \text { (ii) }\left[F_{i}, F_{j}\right] \subset F_{i+j} \\
\text { (iii) } \bigcup_{j} F_{j}=L(X, Y) & \text { (iv) } X \in F_{j} \text { with } j \leq 0 \text { implies } X(0)=0 .
\end{array}
$$

Such filtrations will be constructed from brackets in the free Lie algebra on the symbols $X, Y$ as follows. Assign integer (negative integers permitted) weights to the symbols $X, Y$, denoted wt $X$, wt $Y$. Let the weight of a Lie product be the sum of the weights of its factors and identify any bracket in the free Lie algebra with the same bracket of the vector fields $X, Y$. The weight induced filtration $\mathcal{F}$ then has as subspace $F_{j}$ all elements in $L(X, Y)$ identified with brackets of weight $i \leq j$ in the free Lie algebra. Note that condition (iv) puts a severe restriction on the admissible weights one can assign to $X$ and $Y$.

\subsection{Filtration induced COORDinates and Dilation}

Let $\mathcal{F}=\left\{F_{j}:-\infty<j<\infty\right\}$ be an extended filtration of $L(X, Y)$ at zero and $n_{j}=\operatorname{dim} F_{j}(0),-\infty<j<\infty$. Property (iv) shows $n_{j}=0$ if 
$j \leq 0$ while $\operatorname{dim} L(X, Y)(0)=n$ means $\operatorname{dim} F_{N}(0)=n$ for some integer $N$. Choose $X_{\pi_{1}}, \ldots, X_{\pi_{n_{1}}} \in F_{1}$ such that these are linearly independent at zero. Adjoint $X_{\pi_{n_{1}+1}}, \ldots, X_{\pi_{n_{2}}} \in F_{2}$ such that $X_{\pi_{1}}(0), \ldots, X_{\pi_{n_{2}}}(0)$ are linearly independent and continue in this fashion to get $X_{\pi_{1}}, \ldots, X_{\pi_{n}}$ with

$$
X_{\pi_{i}} \in F_{j}, \quad n_{j-1}+1 \leq i \leq n_{j} .
$$

Let $j \geq 1$ be the smallest integer such that $n_{j} \neq 0$. Choose $r_{i}=j$ for $1 \leq i \leq n_{j}, r_{i}=j+1$ for $n_{j}<i \leq n_{j+1}$, etc. The dilation $\delta_{\varepsilon}^{r}$ with $r=\left(r_{1}, \ldots, r_{n}\right)$ chosen as above is called the filtration induced dilation.

In a specific problem vector fields a re initially given relative to some local coordinates, say $x=\left(x_{1}, \ldots, x_{n}\right)$. Let $\exp \left(t X_{\pi_{i}}\right)(q)$ denote the solution of $\dot{x}=X_{\pi_{i}}(x), x(0)=q$ at time $t$. Define a local coordinate change $y=\varphi^{-1}(x)$ where

$$
x=\varphi(y)=\left(\exp y_{1} X_{\pi_{1}}\right) \circ \cdots \circ\left(\exp y_{n} X_{\pi_{n}}\right)(0) .
$$

Then $\varphi$ is a local diffeomorphism with $\varphi(0))=0$. The coordinates $y=$ $\left(y_{1}, \ldots, y_{n}\right)$ are called the filtration induced coordinates. If $X(x)$ was a vector field given in the original $x$ coordinates, we abuse notation and let $X(y)$ denote this vector field in the new coordinates.

Theorem 2.3. ([12, Thm. 2.1], [18]) Let $\mathcal{F}=\left\{F_{j}:-\infty<j<\infty\right\}$ be an extended filtration at zero with $y=\left(y_{1}, \ldots, y_{n}\right)$ and $\delta_{\varepsilon}^{r}$ the induced local coordinates and dilation. Then if $X \in F_{k}$ (i.e., $w t X=k$ ) it admits the expansion

$$
X(y)=X^{(1-k)}(y)+X^{(2-k)}(y)+\cdots
$$

where $X^{(1-k)}$ is not identically zero.

The construction of filtration induced dilations, coordinates and homogeneous approximating systems will be illustrated by examples to follow. We first describe how to choose the weight assignments for a filtration from system (1.1), in a coordinate free manner. In the figure below (which we call a Kawski diagram) a point of the type $(k, \ell)$ designates brackets of $X, Y$ containing $k$ factors $Y$ and $\ell$ factors $X$.

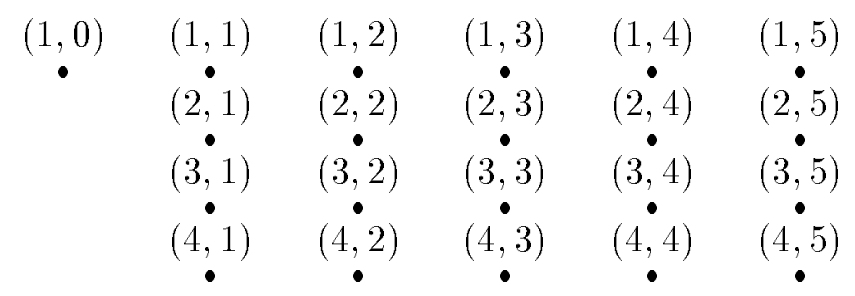

Figure 1.

The main theorem in [22] can be described, via this diagram, as follows. Theorem 2.4. (Sussmann [22]) Suppose one can draw a line through the $(1,0)$ point of the Kawski diagram of slope $k / m$ where $m \geq 1, m \geq k \geq 0$ (i.e., of slope between 0 and 1) such that as one moves the line downward and to the right parallel to itself (or downward if $k=0$ ) any time one reaches a bracket of type (even, odd) which when evaluated at zero is not zero, its value at zero is a linear combination of brackets (at zero) previously reached. Then 
the weight assignment wt $X=k, w t Y=m$ gives a filtration such that in the induced coordinates and relative to the induced dilation, the approximating system is

$$
\dot{y}=X^{(1-k)}(y)+u Y^{(1-m)}(y)
$$

and is STLC at zero. Since either $k=0$ or $k \geq 0$ and $m-k \geq 0$, by theorem 2.1 system (1.1) is STLC at zero.

EXample 2.5. (Sussmann [22]). Let $X(x)=x_{1} \frac{\partial}{\partial x_{2}}+\left(x_{2}^{2}+x_{1}^{3}\right) \frac{\partial}{\partial x_{2}}, Y=\frac{\partial}{\partial x_{1}}$. Then letting $(\operatorname{ad} X, Y)=[X, Y],\left(\operatorname{ad}^{2} X, Y\right)=[X,[X, Y]]$, etc., $(\operatorname{ad} X, Y)(x)$ $=\frac{\partial}{\partial x_{2}}+3 x_{1}^{2} \frac{\partial}{\partial x_{3}},\left(\operatorname{ad}^{2} X, Y\right)(x)=2 x_{2} \frac{\partial}{\partial x_{3}}, W=\left[\left(\operatorname{ad}^{2} X, Y\right),[X, Y]\right]=2 \frac{\partial}{\partial x_{3}}$, $\left(\operatorname{ad}^{3} Y, X\right)=-6 \frac{\partial}{\partial x_{3}}$. Here $W$ is of "bad" bracket type $(2,3)$ thus one wants a line of slope $\mathrm{k} / \mathrm{m}$ such that it gets to the good $(3,1)$ bracket $\left(\operatorname{ad}^{3} Y, X\right)$ before $W$.

(a) Choose our initial line through the $(1,0)$ point to have slope 1 , i.e., $k=m=1$. The parallel translates of this line satisfy theorem 2.4. The weight assignment is wt $X=1$, wt $Y=1$ which gives wt $[X, Y]=2$, wt $\left(\operatorname{ad}^{3} Y, X\right)=4$, wt $W=5$. Then in the filtration $\mathcal{F}, Y=X_{\pi_{1}} \in F_{1}$, $[X, Y]=X_{\pi_{2}} \in F_{2}, F_{3}(0)$ contains no new linearly independent vectors but $\left(\operatorname{ad}^{3} Y, X\right)=X_{\pi_{3}} \in F_{4}$ gives the third and final "direction." The induced dilation is $\delta_{\varepsilon}^{r}$ with $r=(1,2,4)$. The induced coordinates, from $(2.2)$, are easily calculated as $y_{1}=x_{1}, y_{2}=x_{2}, y_{3}=(-1 / 6) x_{3}$. In these coordinates $X(y)=X^{(0)}(y)+X^{(1)}(y)$ where $X^{(0)}(y)=y_{1} \frac{\partial}{\partial y_{2}}-\left(\frac{1}{6}\right) y_{1}^{3} \frac{\partial}{\partial y_{3}}$, $X^{(1)}(y)=y_{2}^{2} \frac{\partial}{\partial y_{2}}$ while $Y(y)=Y^{(0)}(y)=\frac{\partial}{\partial y_{1}}$. The homogeneous approximating system becomes $\dot{y}=X^{(0)}(y)+u Y^{(0)}$, which is STLC at zero.

(b) An initial line of slope $2 / 3$ through the point $(1,0)$ is also such that its parallel translates satisfy theorem 2.4 . Here one would assign wt $X=2$, wt $Y=3$ which gives wt $[X, Y]=5$, wt $\left(\operatorname{ad}^{3} Y, X\right)=11$ and wt $W=12$. The weight generated filtration induces the dilation $\delta_{\varepsilon}^{r}$ with $r=(3,5,11)$. The induced local coordinates are exactly as in part (a) above. In these coordinates, and relative to the induced dilation, the approximating system is $\dot{y}=X^{(-1)}(y)+u Y^{(-2)}$ where $X^{(-1)}(y)=y_{1} \frac{\partial}{\partial y_{2}}-\left(\frac{1}{6}\right) y_{1}^{3} \frac{\partial}{\partial y_{3}}$, and $Y^{(-2)}=$ $\frac{\partial}{\partial y_{2}}$.

Theorem 2.4 handles the case where the approximating system being STLC implies the original system is STLC. We next take the case where the approximating system STLC implies the original system is LTLC.

Theorem 2.6. ([11]) For system (1.1), assume there is an $m \geq 0$ such that the $(1, m+1)$ bracket vanishes at zero (hence this will be true for all $(1, \ell)$ brackets with $\ell \geq(m+1)$ since $X(0)=0)$. If for some integer $k \leq-1$ one can draw a line through the $(1, m)$ point in the Kawski diagram with (negative) slope $k /(1-m k)$ such that:

(a) All brackets to the right of the line vanish at zero. All (even, odd) brackets on the line vanish at zero.

(b) As one slides the line to the left, parallel to itself, each time one encounters a bracket of type (even, odd) which is not zero at zero, it is a linear combination of bracket types (at zero) already encountered. 
Then the weight assignment wt $X=k$, wt $Y=1-m k$ yields the approximating system (in induced coordinates relative to the induced dilation)

$$
\dot{y}=X^{(1-k)}(y)+u Y^{(m k)}(y)
$$

which is STLC at zero and hence, by theorem 2.1, the system (1.1) is LTLC at zero.

Example 2.7. (Hermes, [11]). Let $X(x)=x_{1} \frac{\partial}{\partial x_{2}}+\left(x_{1}^{2}+x_{2}^{3}\right) \frac{\partial}{\partial x_{3}}, Y=\frac{\partial}{\partial x_{1}}$. The relevant brackets are $Y,(\operatorname{ad} X, Y)=\frac{\partial}{\partial x_{2}}+2 x_{1} \frac{\partial}{\partial x_{3}},\left(\operatorname{ad}^{2} Y, X\right)=2 \frac{\partial}{\partial x_{3}}$, and $\left(\operatorname{ad}^{3}[X, Y], X\right)=-6 \frac{\partial}{\partial x_{3}}$. These are, respectively, of types $(1,0),(1,1)$, $(2,1)$ and (3.4). Choose $m=1, k=-2$ which means we consider a line through the $(1,1)$ point with slope $-2 / 3$. This line passes through the "good" $(3,4)$ point and hence as we slide the line to the left parallel to itself, the "direction" of the "bad" $(2,1)$ bracket has already been attained. With $m=1, k=-2$ we assign wt $X=-2$, wt $Y=3$. Then wt $(\operatorname{ad} X, Y)=1$, wt $\left(\operatorname{ad}^{3}[X, Y], X\right)=1$ and the induced dilation $\delta_{\varepsilon}^{r}$ has $r=(1,1,3)$. Choose $X_{\pi_{1}}=\left(\operatorname{ad}^{3}[X, Y], X\right), X_{\pi_{2}}=(\operatorname{ad} X, Y)$, which are both in $F_{1}$, and $X_{\pi_{3}}=Y$, which is in $F_{3}$ of the filtration $\mathcal{F}$. For the induced coordinates, from (2.2) we obtain $x_{1}=y_{3}, x_{2}=y_{2}, x_{3}=2 y_{2} y_{3}-6 y_{1}$ or $y_{1}=\left(-\frac{1}{6}\right) x_{3}+\left(\frac{1}{3}\right) x_{1} x_{2}$, $y_{2}=x_{2}, y_{3}=x_{3}$. Then $X(y)=\left(\left(-\frac{1}{6}\right) y_{2}^{3}+\left(\frac{1}{3}\right) y_{3}^{2}\right) \frac{\partial}{\partial y_{1}}+y_{3} \frac{\partial}{\partial y_{2}}, Y(y)=$ $\left(\frac{1}{3}\right) y_{2} \frac{\partial}{\partial y_{1}}+\frac{\partial}{\partial y_{3}}$. The approximating system is $\dot{y}=X^{(3)}(y)+u Y^{(-2)}(y)$ where

$$
X^{(3)}(y)=\left(-\frac{1}{6}\right) y_{2}^{3} \frac{\partial}{\partial y_{1}}+y_{3} \frac{\partial}{\partial y_{2}}, \quad Y^{(-2)}(y)=\frac{\partial}{\partial y_{3}},
$$

and this system is STLC at zero. The original system is LTLC at zero but not STLC. The $(2,1)$ bracket is an obstruction to STLC, [21].

Our problem now can be roughly stated as follows. For what values $k, m$ does the homogeneous system (2.3) admit a smooth ASFC $u^{*} \in P_{m-k}$, or the homogeneous system (2.4) a smooth ASFC $u^{*} \in P_{1-m k-k}$, with $u^{*}$ an optimal control of a nonlinear regulator problem? We next explain exactly what will be meant by a nonlinear regulator problem for the homogeneous systems $(2.3),(2.4)$.

The cost function will be

$$
C(u)=\int_{0}^{\infty}\left[e u^{s}(t)+h_{\ell-k}^{+}(y(t))\right] d t, \quad e>0
$$

where $s>1$ is rational with even numerator, odd denominator, and $h_{\ell-k}^{+} \in$ $P_{\ell-k}$ is positive definite with $\ell>k$ to be determined. The maximum principle, applied to system (2.3) with cost functional (2.5), (see [13] or [14]) yields the HJB equation

$$
\begin{gathered}
-\gamma\left(\left(Y^{(1-m)} v\right)(y)\right)^{s /(s-1)}+\left(X^{(1-k)} v\right)(y)=-h_{\ell-k}^{+}(y) \\
\gamma=((s-1) / s)(1 / s e)^{1 /(s-1)} \rightarrow \infty \text { as } e \rightarrow 0^{+}
\end{gathered}
$$


for the value function $v$. System (2.4) with cost given by (2.5) has HJB equation

$$
-\gamma\left(\left(Y^{(m k)} v\right)(y)\right)^{s /(s-1)}+\left(X^{(1-k)} v\right)(y)=-h_{\ell-k}^{+}(y) .
$$

If $v \in P_{\ell}$ is a positive definite solution of (2.6) or (2.7), the optimal control, which would be an ASFC, is given, respectively by

$$
\begin{aligned}
& u^{*}(y)=-\gamma\left(\left(Y^{(1-m)} v\right)(y)\right)^{1 /(s-1)} \\
& u^{*}(y)=-\gamma\left(\left(Y^{(m k)} v\right)(y)\right)^{1 /(s-1)},
\end{aligned}
$$

In order that $u^{*}$ be smooth, (2.8) shows it is necessary that either $1<s \leq$ 2 and $(1 /(s-1))=p \geq 1$ be an (odd) integer or $\left(Y^{(1-m)} v\right)(y),\left(Y^{(m k)} v\right)(y)$ have the form $(f(y))^{s-1}$ for $f \in P_{m-k}, f \in P_{1-m k-k}$, respectively, $s \geq 2$ and even. (When $s=2$, the two cases give the same control.) Henceforth, let $p \geq 1$ denote an odd integer related to $s$, when $1<s \leq 2$, by

$$
1 /(s-1)=p, \quad s /(s-1)=p+1 .
$$

Since systems (2.3), (2.4) are STLC at zero, their value functions $v$ for the associated cost (2.5) are continuous, positive definite, and the unique viscosity solutions of $(2.6),(2.7)$, respectively. (See [2], [9].) In general, one cannot expect smooth solutions of $(2.6),(2.9)$ to exist.

\subsection{The Classification Problem}

Assuming there are no stable manifolds for the rest solution of positive dimension for the vector field $X^{(1-k)}$, classify, up to homogeneity preserving feedback and coordinate transformations, systems of the form (2.3), (2.4) which admit a smooth ASFC $u^{*} \in P_{m-k}$, or $u^{*} \in P_{1-m k-k}$ of the form (2.8$2.3),(2.8-2.4)$, respectively, where $v$ is a smooth, positive definite solution of the associated HJB equations (2.6), (2.7).

3. NeCEssary CONDitions that $(2.3),(2.4)$

HAVE A SMOOTH ASFC OF THE FORM $(2.8-2.3),(2.8-2.4)$

The necessary conditions of theorems $3.1,3.5$ of this section depend on the assumption that $X^{(1-k)}$ have no stable manifold of positive dimension. Example 3.4, following the statement of theorem 3.1, illustrates that the conditions are not necessary if this assumption is violated.

\subsection{The case when (1.1) and its approximation (2.3) are STLC}

We begin with the case of system (2.3) with HJB eq. (2.6) and control $u^{*}$ given by $(2.8-2.3)$, with $(1 /(s-1))=p \geq 1$ odd, so $1<s \leq 2$. In order that the ASFC of the approximating system (2.3) be a local ASFC for (1.1), or more specifically that theorem 2.2 applies, we require $u^{*} \in P_{m-k}$. If $v \in P_{\ell}, Y^{(1-m)} v \in P_{\ell-m}$ and $u^{*}$, as given by (2.8-2.3), is in $P_{p(\ell-m)}$. Thus we require $p(\ell-m)=m-k$ or equivalently,

$$
\begin{aligned}
(p+1)(\ell-m)= & (\ell-k) \text { or } \ell=(m-k+p m) / p, \\
& p \geq 1 \text { odd, } m \geq 1, m \geq k \geq 0 .
\end{aligned}
$$


This is also precisely the relationship $k, \ell, m, p$ must satisfy in order that (2.6) admit a dilation group as a symmetry group, i.e., have a solution $v \in P_{\ell}$.

Now for $v$ to be positive definite, $\ell$ must be an even multiple of each $r_{i}, i=1, \ldots, n$. From the filtration construction giving equation (2.3), wt $Y=m \geq 1$, wt $X \geq 0$ implies brackets of $X, Y$ have weights greater than or equal to $m$. $Y(0) \neq 0$ so we can choose $X_{\pi_{1}}=Y$ and $r_{1}=m$. Thus we require $\ell=q m, q \geq 2$ and even, which, in (3.1), gives

$$
m(p-p q+1)-k=0, \quad m \geq 1, p \geq 1, q \geq 2, m \geq k \geq 0 .
$$

But $(p-p q+1) \leq 0$ hence $(3.2)$ can only hold if $p=1, q=2, k=0$, $m \geq 1$ arbitrary. For $p=1, k=0$, eq. (3.1) shows $\ell=2 m$. Since $m=r_{1} \leq r_{2} \leq \cdots \leq r_{n}$ and $\ell$ must be an even multiple of all $r_{i}$, the only solution is $m=r_{1}=r_{2}=\cdots=r_{n}$.

Furthermore, there is no loss in generality in assuming that $m=1$, since this merely scales the $r_{i}$ and leaves them all integers, i.e., we may take $\delta_{\varepsilon}^{r}=\delta_{\varepsilon}^{1}$, the standard dilation.

Next, suppose $\left(Y^{(1-m)} v\right)(y)=(f(y))^{s-1}, s \geq 2$ even, so $u^{*}(y)=-\gamma f(y)$. We require $u^{*} \in P_{m-k}$ or $f(y) \in P_{m-k}$. But, as above, wt $Y=m$, wt $X \geq$ 0 implies $r_{1}=m$. Thus if $k>0, f(y)$ is a constant, hence $k=0$ is necessary and $f(y) \in P_{m}$. But $k=0$ means wt $X=0$ while $Y(0) \neq 0$ means wt $Y=m \geq 1$. If we assign wt $Y=m \geq 2$, all nonvanishing (at zero) brackets will have weights integer multiples of $m$, i.e. all $r_{i}$ will be integer multiples of $r_{1}=m$ and hence, with no loss of generality, we can assume that $m=1$. Suppose $r_{1}=\cdots=r_{j}=1, r_{j+1}, \ldots, r_{n}>1$ with $j<n$. Then wt $Y=1$ implies we can choose $X_{\pi_{1}}=Y$ in the filtration. Since $Y(x)=\frac{\partial}{\partial x_{1}}$, this means $Y(y)=\frac{\partial}{\partial y_{1}}$, Thus $f(y) \in P_{1}$ must have the form $f(y)=\sum_{i=1}^{j} \alpha_{i} y_{i}$. Also, $\left(Y^{(0)} v\right)(y)=v_{y_{1}}(y)=(f(y))^{s-1}, s \geq 2$ even, means $v(y)=\left(1 / \alpha_{1} s\right)(f(y))^{s}+g\left(y_{j+1}, \ldots, y_{n}\right)$ where $g \in P_{s}$ is a positive definite function. Now system $(2.3)$ has $k=0, m=1$ and control $u^{*}(y)=$ $-\gamma f(y)$ which means the right sides of the first $j$ equations in the controlled system are linear functions of $y_{1}, \ldots, y_{j}$. Then for the right side of $(2.6)$ to be negative definite it is necessary that $\left(X^{(1)} g\right)\left(0, \ldots, 0, y_{j+1}, \ldots, y_{n}\right)$ be a negative definite function of $y_{j+1}, \ldots, y_{n}$. But if this were the case, the solution, $x(t, c)$, of $\dot{x}=X^{(1)}, x(0)=c \neq 0$ but $c_{1}, \ldots, c_{j}=0$, would satisfy $x(t, c) \rightarrow 0$ as $t \rightarrow \infty$. This contradicts the assumption that the rest solution zero of the uncontrolled system has no stable manifold of positive dimension. Thus $j=n$ and $r_{1}, \ldots, r_{n}=1$.

Geometrically, $k=0$ means the line in theorem 2.4 has slope zero, while all $r_{i}=m$ means dimspan $\left\{Y^{(1-m)}(0), \ldots,\left(\operatorname{ad}^{n-1} X^{(1-k)}, Y^{(1-m)}\right)(0)\right\}=n$.

With $k=0, m=1$, in the latter case we may choose $s=\ell=2$, while in the former $q=2$ so we have $\ell=m q=2$ while $p=1$ means $s=2$. Thus the cost (2.5) involves $u^{2}$ while $h_{\ell-k}^{+}=h_{2}^{+}$is a positive definite quadratic form. Here $X^{(1-k)}(y)=X^{(1)}(y)$ which, relative to the standard dilation $\delta_{\varepsilon}^{1}$, means linear in $y, Y^{(1-m)}=Y^{(0)}$ while $\operatorname{dim}\left\{Y^{(0)}(0), \ldots,\left(\operatorname{ad}^{n-1} X^{(1)}, Y^{(0)}\right)(0)\right\}=$ $n$ means system (2.3) is a controllable linear system. The optimization problem is the standard "linear, quadratic, regulator problem." Classical 
theory, e.g. [17], shows that for any $h_{2}^{+} \in P_{2}$ the HJB equation (2.6) has a positive definite solution $v \in P_{2}$ (i.e., a quadratic form) and the ASFC $u^{*}$ has the form given in (2.8-2.3). The above can be summarized as

THEOREM 3.1. In order that the homogeneous system (2.3), (which is assumed to have no stable manifold of positive dimension for the zero solution of the uncontrolled system and to be STLC at zero) admit a homogeneous ASFC $u^{*} \in P_{m-k}$ of the form (2.8-2.3), where $v$ is the value function for a regulator problem, it is necessary and sufficient that $k=0, m=1$, and homogeneity be relative to the standard dilation, i.e. $Y(0),(\operatorname{ad} X, Y)(0), \ldots$, $\left(a d^{n-1} X, Y\right)(0)$ be linearly independent in system (1.1).

REMARK 3.2. In essence, the above states that if the system (1.1) is STLC at zero and has a homogeneous approximation of the form (2.3) which admits an ASFC of the form (2.8-2.3), the approximation has to be a controllable linear system and we are in the case of a linear, quadratic, regulator for (2.3). Also the group of homogeneity preserving transformations consists of the linear coordinate and linear feedback transformations. A "nice" canonical representative for the equivalence class of the controllable linear system is the "first order integrator" $\dot{y}_{1}=u, \dot{y}_{2}=y_{1}, \ldots, \dot{y}_{n}=y_{n-1}$.

REMARK 3.3. The group consisting of linear feedback and linear coordinate changes is a subgroup of the "feedback group" of arbitrary (smooth) feedback and coordinate transformations used in the problem of feedback linearization. With dilation the standard dilation $\delta_{\varepsilon}^{1}$, the homogeneous approximation (2.3) of system (1.1) is the standard linearization. One may note that if (1.1) is feedback linearizable, its linear approximation will be a controllable, linear system.

EXAMPLE 3.4. This example is to illustrate the reason for the assumption that the zero solution of the uncontrolled system have no stable manifold of positive dimension. Let

$$
X(y)=y_{1} \frac{\partial}{\partial y_{2}}+\left(y_{2}^{3}-y_{3}\right) \frac{\partial}{\partial y_{3}}, \quad Y=\frac{\partial}{\partial y_{1}}
$$

Then $Y(0),(\operatorname{ad} X, Y)(0), W(0)=\left(\operatorname{ad}^{3}[X, Y], X\right)(0)$ are linearly independent. Also, $\left(\operatorname{ad}^{k} X, W\right)(0) \neq 0$ for all $k \geq 0$ so one cannot assign wt $X<0$ without violating property $(i v)$ of a filtration at zero. Assign wt $Y=1$, wt $X=0$ which yields $\delta_{\varepsilon}^{r}, r=(1,1,3)$ and $X=X^{(1)}, Y=Y^{(0)}$ with homogeneity relative to $\delta_{\varepsilon}^{r}$. The rest solution, zero, of the uncontrolled system does admit the stable, one dimensional, manifold $\left\{x \in \mathbb{R}^{3}: x_{1}=x_{2}=0\right\}$ ! The necessary conditions to theorem 3.1 are not satisfied, but here one can choose $\ell=s=6$ which gives, as left side of $(2.6),-\gamma\left(v_{y_{1}}(y)\right)^{6 / 5}+y_{1} v_{y_{2}}(y)+$ $\left(y_{2}^{3}-y_{3}\right) v_{y_{3}}$. For $c, \gamma>0$ sufficiently large, the smooth, positive definite function $v(y)=(1 / 6)\left(3 y_{1}+2 y_{2}\right)^{6}+y_{2}^{6}+c y_{3}^{2} \in P_{6}$ makes the preceding expression negative definite, i.e. equal to some $-h_{6}^{+} \in P_{6}$. This $v$ is a smooth solution of an HJB equation of the form (2.6) and $u^{*}(y)=-\gamma\left(v_{y_{1}}(y)\right)^{1 / 5}=$ $-\gamma\left(3 y_{1}+2 y_{2}\right)$ is a smooth ASFC in $P_{1}$ (as can easily seen by linearization) which does not, and need not, depend on $y_{3}$, a variable which tends to zero with no control. 


\subsection{The Case When (1.1) Is LTLC WITH HOMOGENEOUS APPROXIMATION (2.4)}

Again, it is necessary to consider two cases; $1 /(s-1)=p \geq 1$ odd, i.e. $1<s \leq 2$ and $s \geq 4$ even. We begin with:

NECESSARY CONDITIONS WHEN $1<s \leq 2$. Here $p$ and $s$ are related as in (2.9). In order that theorem 2.2 can be applied to show an ASFC $u^{*}$ for system (2.4) is a local ASFC for system (1.1), it is required that $u^{*} \in$ $P_{1-k-m k}$. If $v \in P_{\ell}, Y^{(m k)} v \in P_{\ell+m k-1}$ and $u^{*}$, as given by (2.8-2.4), is in $P_{p(\ell+m k-1)}$. Thus it is necessary that

$$
\begin{aligned}
& p(\ell+m k-1)=1-k-m k, \quad p \geq 1 \text { odd integer } \\
& m \geq 0, k \leq-1, \ell \geq 2 \text { even }
\end{aligned}
$$

Also, for (2.7) to admit a dilation group as a symmetry group, for $v \in P_{\ell}$ one needs $\left(Y^{(m k)} v\right)^{p+1} \in P_{\ell-k}$ or $(p+1)(m k+\ell-1)=\ell-k$, and this is satisfied if $k, \ell, m, p$ satisfy (3.3). Furthermore, for $v$ to be positive definite, $\ell$ must be an even multiple of each $r_{i}, i=1, \ldots, n$. Our original coordinate choice $Y(x)=\frac{\partial}{\partial x_{1}}$ and $X(x)=\sum_{j=2}^{n} a_{j}(x) \frac{\partial}{\partial x_{j}}$ means the direction $Y(0)$ cannot occur as any Lie product of $X, Y$ evaluated at zero, hence the coordinate change (2.2) induced by the filtration, has $Y=X_{\pi_{i}}$ for some $i=1, \ldots, n$. This means $r_{i}=$ wt $Y=1-m k$ and thus it is necessary that

$$
\ell=q(1-m k) \text { for some } q \geq 2 \text { and even. }
$$

Also, in order that each side of $(2.7)$ be a negative definite, homogeneous polynomial in $P_{\ell-k}$, it is necessary that $(\ell-k)$ be an even multiple of each $r_{i}, i=1, \ldots, n$.

LeMma 3.5. When $1 /(s-1)=p \geq 1$ odd, necessary conditions that system (2.4) admit an $A S F C u^{*} \in P_{1-k-m k}$ of the form (2.8-2.4) are that (3.3), (3.4) have a simultaneous solution and $\ell, \ell-k$ be even multiples of all $r_{i}$. This occurs only if $m=0, k=-(p q-p-1) \leq-1, p \geq 1$ odd, $q \geq 2$ even, $\ell=q$ and both $q$ and $(q-k)$ are even multiples of all $r_{i}$.

Proof. Substituting for $\ell$ from (3.4) into (3.3) gives

$$
-k[m(p q-p-1)-1]=-(p q-p-1) .
$$

But $(p q-p-1) \geq 0$. If $(p q-p-1)=0,(3.5)$ yields $k=0$, which is not valid. Suppose $(p q-p-1) \geq 1$. If $m=1$, the left side of $(3.5)$ is zero, the right side negative. If $m \geq 2$, since $k \leq-1$ the left side of (3.5) is positive, the right side negative. This leaves only the case $m=0, k=-(p q-p-1) \leq-1$.

It is interesting, and instructive, to see by example how severe the conditions of lemma 3.5 are on possible systems.

Example 3.6. (An example which satisfies all necessary conditions of lemma 3.5, but does not satisfy necessary condition (c) of theorem 3.8 below.) Lemma 3.5 requires $m=0, \ell=q$, hence $k=-(p \ell-p-1)$, $\ell-k=(1+p)(\ell-1)$ and these must both be even multiples of all $r_{i}$. If for integers $n_{i}, m_{i}>n_{i}$, since $k \leq-2,(1+p)(\ell-1)=2 m_{i} r_{i}, \ell=2 n_{i} r_{i}$ 
then $r_{i}=(1+p) / 2\left(n_{i}(1+p)-m_{i}\right)$ and it follows that $1 \leq r_{i} \leq(1+p) / 2$, $i=1, \ldots, n$. If, for example, we want $r=(1,2,3,3)$, the smallest $p=5$ and $r_{2}=r_{3}=3$ is attained with $n_{3}=1, m_{3}=5$. Then the smallest possible $\ell$ is $\ell=6$ which means $k=24$. For example, a system on $\mathbb{R}^{4}$, such as

$$
X(y)=\left(y_{1}^{26}-y_{2} y_{3}^{8}\right) \frac{\partial}{\partial y_{2}}+\left(y_{1}^{27}-y_{3}^{9}+y_{4}^{9}\right) \frac{\partial}{\partial y_{3}}+\left(y_{1}^{27}+y_{3}^{9}-y_{4}^{9}\right) \frac{\partial}{\partial y_{4}}, \quad Y=\frac{\partial}{\partial y_{1}}
$$

satisfies the conditions of lemma 3.5 .

It is instructive to note, for a step in the proof of theorem 3.8, that since $r_{2}<r_{3}$ and $k$ is an integer multiple of all $r_{i}$, the coefficient of $\frac{\partial}{\partial x_{2}}$ cannot contain a term $c y_{3}^{n_{i}}$. Indeed this would require both $r_{3}+k$ and $r_{2}+k$ to be integer multiples of $r_{3}$.

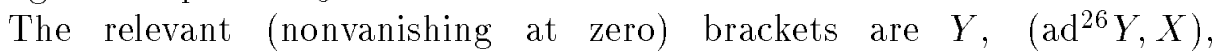
$\left(\operatorname{ad}^{27} Y, X\right),\left(\operatorname{ad}^{9}\left(\operatorname{ad}^{27} Y, X\right)\right)$ which are, respectively, of types $(1,0),(26,1)$, $(27,1),(243,10)$. Assign wt $X=-24=k$, wt $Y=1$. Then the above brackets have, respectively, weights $1,2,3$, and 3 , and $\delta_{\varepsilon}^{r}$ with $r=(1,2,3,3)$, as desired. Thus all conditions of lemma 3.5 are satisfied. Also $X(y)=$ $X^{(25)}(y)$ is homogeneous of degree 25 with respect to $\delta_{\varepsilon}^{r}$. One would seek an ASFC $u^{*} \in P_{25}$. Since $\delta_{\varepsilon}^{r} \neq \delta_{\varepsilon}^{1}$, the system $\dot{x}=X(x)+u Y$ does not satisfy condition (c) of theorem 3.8 , below, and hence does not admit a smooth ASFC $u^{*} \in P_{25}$ of the form (2.8-2.4).

REMARK 3.7. The condition $m=0$ means $Y^{(m k)}=Y^{(0)}$ and $\left(\operatorname{ad} X^{(1-k)}\right.$, $\left.Y^{(0)}\right)(0)=0$ in the homogeneous approximating system (2.4). Also, $m=0$ means wt $Y=1$ and in theorem 2.6 , brackets of type $(-k a+1, a), a=$ $0,1, \ldots$, on the line through the $(1,0)$ point with slope $k \leq-1$ will have weight 1 . If there are $n$ linearly independent brackets (evaluated at zero) on this line, one will have $r_{1}=r_{2}=\cdots=r_{n}=1$. If not, as the line is moved to the left parallel to itself, linearly independent directions will occur from brackets of weight $\geq 2$ and there will be some $r_{i} \geq 2$.

Theorem 3.8. Necessary conditions that the homogeneous system (2.4), (which is assumed to have no stable manifold of positive dimension for the zero solution of the uncontrolled system) admit a homogeneous ASFC $u^{*} \in P_{1-m k-k}, m \geq 0, k \leq-1$, of the form (2.8-2.4) are: (a) $k \in$ $\{-2,-4,-6, \ldots\}$, (b) $m=0$, (c) homogeneity, in (2.4), be relative to the standard dilation $\delta_{\varepsilon}^{1}$, i.e. n linearly independent (at zero) brackets of $X^{(1-k)}$, $Y^{(0)}$ lie on the line of slope $k$ through the point $(1,0)$ in figure 1.

REMARK 3.9. One can summarize the essence of theorems 3.1, 3.5 as follows. In order that the system (1.1) have a homogeneous approximation of either the form (2.3) or (2.4) which admits a smooth ASFC $u^{*}$ of the form (2.8$2.3)$ in $P_{m-k}$ or of the form $(2.8-2.4)$ in $P_{1-m k-k}$, it is necessary that one can assign wt $Y=1$, wt $X=k, k \in\{0,-2,-4, \ldots\}$ and have $n$ linearly independent (at zero) brackets of $X, Y$ of weight one, i.e. these lie on the line through $(1,0)$ with slope $k$, in figure 1 . (Note that this will, of necessity, imply that these are good, i.e. not (even, odd) types of brackets, which means the approximating system will be STLC.)

Proof. (a) We first deal with the case $1 /(s-1)=p \geq 1$ odd, or $1<s \leq 2$. Lemma 3.5 shows that $m=0$ is necessary, so $Y^{(m k)}=Y^{(0)}$ which means 
wt $Y=1$ and in the construction of local coordinates from the filtration, we can choose $X_{\pi_{1}}(x)=Y(x)=\frac{\partial}{\partial x_{1}}$ which means $Y(y)=\frac{\partial}{\partial y_{1}}$ and $Y^{(0)}=\frac{\partial}{\partial y_{1}}$. Also, $\ell=q$ and $k=-(p \ell-p-1) \leq 1, p \geq 1$ odd, forces $k$ to be even, i.e. $k \in\{-2,-4, \ldots\}$. This means in figure 1 we consider a line through the $(1,0)$ point with slope $k \leq-2$. Now wt $Y=1=r_{1} \leq r_{2} \leq \cdots \leq r_{n}$. Lemma 3.10, which follows, will show that it is necessary that all $r_{i}=1$, finishing the proof of (a).

Lemma 3.10. If $m=0$, which implies $Y^{(0)}=\frac{\partial}{\partial y_{1}}$, and $X^{(1-k)}$ has no stable manifold for the zero solution of positive dimension, it is necessary that all $r_{i}=1, i=1, \ldots, n$.

PROOF. Suppose $r_{n}>1$, or specifically, $1=r_{1} \leq r_{2} \leq \cdots \leq r_{j}<r_{j+1}=$ $\cdots=r_{n}$. Then for $1 \leq i \leq j, v$ cannot contain a term $c y_{i} y_{j+1}^{m_{j+1}} \ldots y_{n}^{m_{n}}$ with $c \neq 0, m_{j+1}, \ldots, m_{n} \geq 0$. Indeed, if it did, $r_{i}+r_{n}\left(m_{j+1}+\cdots+m_{n}\right)=l$. But $\ell$ is an even multiple of $r_{n}$ hence dividing the above equality by $r_{n}$ and using $r_{n}>r_{i}$ gives a contradiction. Thus we conclude

$$
\left(Y^{(0)} v\right)\left(0, \ldots, 0, y_{j+1}, \ldots, y_{n}\right)=v_{y_{1}}\left(0, \ldots, y_{j+1}, \ldots, y_{n}\right)=0 .
$$

Also, if $X^{(1-k)}(y)=\sum_{j=2}^{n} a_{j}(y) \frac{\partial}{\partial y_{j}}$ then for $2 \leq i \leq j$,

$$
v_{y_{i}}\left(0, \ldots, 0, y_{j+1}, \ldots, y_{n}\right) a_{i}\left(0, \ldots, 0, y_{j+1}, \ldots, y_{n}\right)=0 .
$$

(One can also note that $a_{i} \in P_{r_{i}+k}$ hence it cannot contain a term $y_{i+1}^{m_{j+1}} \ldots y_{n}^{m_{j}}$ since this would require both $r_{i+k}$ and $r_{n}+k$ to be integer multiples of $r_{n}>r_{i}$. This means $a_{i}\left(0, \ldots, 0, y_{j+1}, \ldots, y_{n}\right)=0$ by itself.)

From (i), (ii), for the left side of (2.7) to be negative definite on the subspace $y_{1}=\cdots=y_{j}=0$ it is necessary that $\left(X^{(1-k)} v\right)$ be negative definite on this subspace. If $j<n$ this is a subspace of positive dimension for the zero solution of $X^{(1-k)}$. Since we assume no such exists, $j=n$, i.e. $1=r_{1}=\cdots=r_{n}$.

(b) Next, suppose $\left(Y^{(m k)} v\right)(y)=(f(y))^{s-1}, s \geq 2$ and even. We require $u^{*} \in P_{1-m k-k}$, but $u^{*}=-\gamma f$ so $f \in P_{1-m k-k}, m \geq 0, k \leq-1$, and $(f(y))^{s} \in P_{s(1-m k-k)}$. For homogeneity in the HJB equation this must also be in $P_{\ell-k}$, i.e. it is necessary that $s(1-m k-k)=\ell-k, k \leq-1, m \geq 0$, $s \geq 2$ even, $\ell$ even. This forces $k$ to be even.

In (1.1), we had $Y=\frac{\partial}{\partial x_{1}}$ and the first component of $X$ was zero so the "direction" $\frac{\partial}{\partial x_{1}}$ cannot occur as a product of $X, Y$, evaluated at zero. This means $Y=X_{\pi_{i}}$ for some $1 \leq i \leq n$, in the change to induced coordinates. Thus wt $Y=1-m k=r_{i}$ for some $1 \leq i \leq n$.

Again, both $\ell$ and $\ell-k$ must be even multiples of all $r_{i}$, which means $-k$ is an even multiple of all $r_{i}$. In particular, $-k=2 n_{1}(1-m k)$ for some integer $n_{1}$, i.e. $k\left(2 m n_{1}-1\right)=2 n_{1}, k \leq-2, m \geq 0$. This forces $m=0$.

We now have $k \leq-2$ even, $m=0$ so wt $Y=1=r_{1} \leq r_{2} \leq \cdots \leq r_{n}$ and $\ell, \ell-k$ must be even multiples of all $r_{i}$. The conclusion that all $r_{i}=1$ follows from lemma 3.10 .

Example 3.11. (The $p$ th power integrator, $p \geq 3$ and odd.) On $\mathbb{R}^{n}$, consider the system

$$
\begin{aligned}
& \dot{y}_{1}=u, \dot{y}_{2}=y_{1}^{p}, \ldots, \dot{y}_{n}=y_{n-1}^{p}, \\
& \text { i.e., } X(y)=y_{1}^{p} \frac{\partial}{\partial y_{2}}+\cdots+y_{n-1}^{p} \frac{\partial}{\partial y_{n}}, Y=\frac{\partial}{\partial y_{1}} .
\end{aligned}
$$


The brackets of interest are $Y(0),\left(\operatorname{ad}^{p} Y, X\right)(0),\left(\operatorname{ad}^{p}\left(\operatorname{ad}^{p} Y, X\right), X\right)(0)$, etc., which are of type $(1,0),(p, 1),\left(p^{2}, p+1\right),\left(p^{3}, p^{2}+p+1\right)$, etc. With the weight assignments wt $Y=1$, wt $X=1-p$, all these bracket types lie on the line (see figure 1 ) through the $(1,0)$ point with slope $k=1-p$, so all have weight 1 . Then $r_{1}=\cdots=r_{n}=1$, the dilation is the standard dilation $\delta_{\varepsilon}^{1}, X(y)=X^{(p)}(y)$ is homogeneous of degree $p$ with respect to $\delta_{\varepsilon}^{1}, Y=Y^{(0)}$, and (3.6) is an STLC homogeneous system of the form (2.4). Here $m=0$, $q=\ell=2, p \geq 3$ odd and $k=1-p$ provide a solution of proposition 3.14.

Proposition 3.12. ([8]) The pth power integrator, system (3.6), with $p \geq 3$ odd, admits an ASFC $u^{*}(y)=-\gamma((Y v)(y))^{p} \in P_{p}, \gamma>0$, where $v(y)=$ $(1 / 2) y^{T} Q y, Q$ positive definite, is a solution of an associated HJB equation (2.7).

Proof. (See appendix 1 for a proof which differs from that outlined in [8].)

REMARK 3.13. If one adds a small homogeneous perturbation to the vector field $X$ in (3.6), i.e. replace $X=X^{(p)}$ by $X^{(p)}+\varepsilon W^{(p)}$ with $W^{(p)}$ homogeneous of degree $p$ with respect to $\delta_{\varepsilon}^{1}$, then for $\varepsilon>0$ and sufficiently small the control $u^{*}$ of proposition 3.12 remains an ASFC for the perturbed system. Indeed, $v$ is a homogeneous Lyapunov function for the controlled system (3.6), with trajectory derivative $\dot{v}<0$ on the compact unit sphere $S^{n-1} \subset \mathbb{R}^{n}$. Thus for $\varepsilon>0$ small, $\dot{v}<0$ on $S^{n-1}$ for the perturbed system, and by homogeneity, $\dot{v}$ is negative definite.

If the restriction that the ASFC be homogeneous is removed, the proof of proposition 3.12, with no essential changes, yields a proof of

Proposition 3.14. Let $p_{1}, \ldots, p_{n-1} \geq 1$ be odd integers. Then for any odd integer $p \geq 1$, the system

$$
\dot{y}_{1}=u, \quad \dot{y}_{2}=y_{1}^{p_{1}}, \ldots, \dot{y}_{n}=y_{n-1}^{p_{n}-1}
$$

admits an $A S F C$ of the form $u^{*}(y)=-\gamma\left(v_{y_{1}}(y)\right)^{p}$ where $v(y)=(1 / 2) y^{T} Q y$ with $Q$ positive definite. However, $u^{*}$ need not be homogeneous.

System (3.7), and system (3.9) of proposition 3.20 , can readily be transformed to "triangular systems" with the coordinate change given in the proof of proposition 3.12. Results on continuous ASFC for triangular systems can be found in [23].

Example 3.15 . On $\mathbb{R}^{3}$, consider the system

$$
\dot{y}_{1}=u, \quad \dot{y}_{2}=y_{1}, \quad \dot{y}_{3}=y_{2}^{3}, \text { i.e. } X(y)=y_{1} \frac{\partial}{\partial y_{2}}+y_{2}^{3} \frac{\partial}{\partial y_{3}}, \quad Y=\frac{\partial}{\partial y_{1}} \text {. }
$$

Relative to the dilation $\delta_{\varepsilon}^{r}, r=(1,1,3), X(y)=X^{(1)}(y)$, i.e., is homogeneous of degree 1 while $Y=Y^{(0)}$. This is a system of the form (1.1-2.3) with $k=0$, $m=1$. By theorem 3.8, it does not admit an ASFC $u^{*} \in P_{1}$ of the form (2.8-2.3). However, by proposition 3.14, it does admit a linear ASFC $u^{*}$ of the form $u^{*}(y)=-\gamma(Y v)(y)$, with $v$ a positive definite quadratic form. However $u^{*}$ is not homogeneous with respect to $\delta_{\varepsilon}^{r}$.

REMARK 3.16. For further work on homogeneous, affine systems which admit an ASFC but do not admit a homogeneous ASFC, see [20]. 
EXAmple 3.17. (Cubic integrator on $\mathbb{R}^{3}$.) Let $X(y)=y_{1}^{3} \frac{\partial}{\partial y_{2}}+y_{2}^{3} \frac{\partial}{\partial y_{3}}$, $Y=\frac{\partial}{\partial y_{1}}$. Then $Y(0),\left(\operatorname{ad}^{3} Y, X\right)(0)$, and $\left(\operatorname{ad}^{3}\left(\operatorname{ad}^{3} Y, X\right), X\right)(0)$ are linearly independent and of types $(1,0),(3,1),(9,4)$ respectively. Theorem 2.6 applies with $m=0, k=-2$, i.e., the line through the point $(1,0)$ with slope -2 is as desired. The weight assignments wt $X=-2$, wt $Y=0$ shows each of the above brackets has weight one, so $\delta_{\varepsilon}^{r}$ has $r=(1,1,1)$, while $X(y)=X^{(3)}(y), Y=Y^{(0)}$ are homogeneous of the indicated degrees with respect to $\delta_{\varepsilon}^{r}$. Theorem 3.8 applies but lemma 3.5 gives the sharper condition $-k=2=(p q-p-1)$. The possible values of $p, q$ are then $p=3, q=2$ or $p=1, q=4$. For $p=3, q=2$ one would have $v \in P_{2}$ (a positive definite, quadratic form) and proposition 3.12 assures the existence of an ASFC $u^{*} \in P_{3}$ of the form $u^{*}(y)=-\gamma((Y v)(y))^{3}$. For $p=1, q=4$ one would have $v \in P_{4}$ and seek an ASFC of the form $u^{*}(y)=-\gamma(Y v)(y)$. The existence of such a $v \in P_{4}$ is an open question.

REMARK 3.18. In the case of the linear regulator, or $p=1$ in system (3.6), the associated HJB equation (2.6) becomes $-\gamma\left(v_{y_{1}}(y)\right)^{2}+\sum_{j=2}^{n} y_{j-1} v_{y_{j}}(y)=$ $-h_{2}^{+}(y)$ and this has a positive definite solution $v \in P_{2}$ for arbitrary, positive definite, quadratic form $h_{2}^{+} \in P_{2}$. For odd $p \geq 3$, this is no longer the case! Indeed, consider the case $n=2, p=3$ in (3.6). The HJB equation (2.7) becomes $-\gamma\left(v_{y_{1}}(y)\right)^{4}+y_{1}^{3} v_{y_{2}}(y)=-h_{4}^{+}(y)$. Suppose one chooses $h_{4}^{+}(y)=$ $y_{1}^{4}+y_{2}^{4}$. Then the most general, positive definite $v \in P_{2}$ has the form $v(y)=a y_{1}^{2}+b y_{1} y_{2}+c y_{2}^{2}, a, c>0$ and $a c-b^{2} / 4>0$. Substituting into the HJB equation with $h_{4}^{+}$as above readily shows one cannot solve for $a, b, c$ (subject to the above restrictions) to yield a solution. Here a positive, definite solution $v$, in the viscosity sense, will exist for any $h_{4}^{+} \in P_{4}$, but $v \in P_{2}$ only occurs for special $h_{4}^{+} \in P_{4}$.

The purpose of the next example is to illustrate that the necessary conditions of theorem 3.8 for the existence of a smooth ASFC of the form (2.8-2.4) are by no means sufficient.

Example 3.19. (Coron [5]). On $\mathbb{R}^{3}$, let $Y=\frac{\partial}{\partial y_{3}}, X(y)=\left(y_{2}^{3}-3\left(y_{1}-\right.\right.$ $\left.\left.y_{3}\right)^{2} y_{2}\right) \frac{\partial}{\partial y_{1}}+\left(\left(y_{1}-y_{3}\right)^{3}-3\left(y_{1}-y_{2}\right) y_{2}^{2}\right) \frac{\partial}{\partial y_{2}}$.

Then $Y(0),\left(\operatorname{ad}^{3} Y, X\right)(0),\left[\left(\operatorname{ad}^{2} Y, X\right),\left(\operatorname{ad}^{3} Y, X\right)\right](0),\left(\operatorname{ad}^{3}\left(\operatorname{ad}^{3} Y, X\right), X\right)(0)$ are the relevant, nonzero brackets, they span $\mathbb{R}^{3}$, and are, respectively, of types $(1,0),(3,1),(5,2),(9,4)$. Theorem 2.6 applies with $m=0, k=-2$ and all four of the above points lie on the line through the point $(1,0)$ with slope -2 in figure 1 . The weight assignment wt $X=-2$, wt $Y=1$ shows $X(y)=X^{(3)}(y), Y=Y^{(0)}$ relative to the standard dilation $\delta_{\varepsilon}^{r}, r=(1,1,1)$. Then, as in example 3.11, the choices $p=3, q=2$ giving $\ell=2$ or $p=1$, $q=4, \ell=4$ may be used in lemma 3.5 and the necessary conditions of theorem 3.8 are satisfied. This example satisfies the Brockett necessary conditions, [4], but not the Coron necessary condition, [5], for the existence of a continuous ASFC. Thus for $p=3, q=2$ there is no $v \in P_{2}$ such that $u^{*}(y)=-\gamma((Y v)(y))^{3}$ is an ASFC or for $p=1, q=4$, there is no $v \in P_{4}$ such that $u^{*}(y)=-\gamma(Y v)(y)$ is an ASFC. For example, with $p=1, q=4$, this means the HJB equation (9), i.e. $-\gamma\left(\left(Y^{(0)} v\right)(y)\right)^{2}+\left(X^{(3)} v\right)(y)=-h_{6}^{+}(y)$ has no positive definite solution $v \in P_{4}$ for any positive definite $h_{6}^{+} \in P_{6}$ 
but for every $h_{6}^{+}$, it does have a positive definite viscosity solution $v$. (See $[2],[9]$.

\section{3. $\delta_{\varepsilon}^{r}$ FeEdBack equivalence}

Let $W^{(k)}(x)=\sum_{j=1}^{n} a_{j}(x) \frac{\partial}{\partial x_{j}}$ be a vector field, homogeneous of degree $k$ with respect to a dilation $\delta_{\varepsilon}^{r}$, in the local coordinates $x$ for $\mathbb{R}^{n}$. A diffeomorphism $\varphi: \mathbb{R}^{n} \rightarrow \mathbb{R}^{n}$, say $x=\varphi(y), \varphi(y)=\left(\varphi_{1}(y), \ldots, \varphi_{n}(y)\right)$ transforms $W^{(k)}(x)$ into $\varphi_{*}^{-1}(y) W^{(k)}(\varphi(y))$, which we denote by $\left(T_{\varphi} W^{(k)}\right)(y)$. A necessary and sufficient condition that $\varphi$ "preserve homogeneity," i.e. that $\left(T_{\varphi} W^{(k)}\right)(y)$ be homogeneous of degree $k$ with respect to $\delta_{\varepsilon}^{r}$ in the new $y$ coordinates is that $\varphi_{i} \in P_{r_{i}}, i=1, \ldots, n$. (See [1] for this and further such results.) Thus the $\delta_{\varepsilon}^{r}$ feedback equivalence group for a system $\dot{y}=X^{(1-k)}(y)+u Y^{(0)}(y)$, where homogeneity is relative to $\delta_{\varepsilon}^{r}$, consists of coordinate changes by diffeomorphisms $y=\varphi(x)=\left(\varphi_{1}(x), \ldots, \varphi_{n}(x)\right)$ with $\varphi_{i} \in P_{r_{i}}$ and feedback $u=\alpha(y)+\beta(y) \mu$ where $\alpha \in P_{1-k}, \beta \in P_{0}$ and $\mu$ is a new control. Theorem 3.1 shows that a necessary condition for system (2.4) to admit an ASFC $u^{*}$ of the form (2.8-2.4) is that $\delta_{\varepsilon}^{r}=\delta_{\varepsilon}^{1}$. The diffeomorphism subgroup which preserves homogeneity relative to $\delta_{\varepsilon}^{1}$ is just the group of linear diffeomorphisms, i.e. $x=M y, M \in G \ell(n, \mathbb{R})$. The following questions now arise. What are the $\delta_{\varepsilon}^{1}$ feedback equivalent systems to the odd power integrators? Are there systems which satisfy the necessary conditions of theorem 3.1 which are not in such an equivalence class? Do any such systems actually admit a smooth ASFC of the form (2.8-2.4)?

Let $X(y), Y=\frac{\partial}{\partial y_{1}}$ be the vector fields for $p$ th power integrators given in (3.6), $p \geq 3$ odd, $M \in G \ell(n, \mathbb{R})$ and $y=\varphi(x)=M x$ the coordinate diffeomorphism. The systems which are in the $\delta_{\varepsilon}^{1}$ feedback equivalence class of (3.6) have the form $\dot{x}=V(x)+\mu W$ where

$$
V(x)=M^{-1}\left[X(M x)+u_{p}(M x) Y\right], W=c M^{-1} Y, c \neq 0, u_{p} \in P_{p}
$$

and $\mu$ a new control. The final two questions, above, are answered by

Proposition 3.20. Let $p \geq 3$ be odd, a,e $\geq 1$, with a odd, e even, such that $a+e=p$. We consider the system with

$$
V(x)=\left(x_{1}^{p}+x_{1}^{a} x_{2}^{e}\right) \frac{\partial}{\partial x_{2}}+\cdots+\left(x_{n-1}^{p}+x_{n-1}^{a} x_{n}^{e}\right) \frac{\partial}{\partial x_{n}}, \quad Y=\frac{\partial}{\partial x_{1}} .
$$

System (3.9) is not in the $\delta_{\varepsilon}^{1}$ feedback equivalence group of the pth power integrator (3.6). System (3.9) admits an ASFC of the form (2.8-2.4) where $v(x)=(1 / 2) x^{T} Q x, Q$ positive definite, is a solution of an HJB equation of the form (2.7).

Proof. Clearly $V$ is homogeneous of degree one with respect to $\delta_{c}^{1}, Y$ is homogeneous of degree zero. To show (3.9) is not in the $\delta_{\varepsilon}^{1}$ feedback equivalence class of (3.6), it suffices to take the case $n=2$. With $X, Y$ as given in (3.6), calculation using (3.8) easily shows there is no $M \in G \ell(2, \mathbb{R})$, $c \neq 0$ and $u_{p} \in P_{p}$ such that one can transform system (3.6) to system (3.9). The proof that system (3.9) admits an ASFC of the form (2.8-2.4) with $v(x)=(1 / 2) x^{T} Q x$ is given in appendix 1 , as remark $\mathrm{A} 1$, following the proof of proposition 3.12 . 


\section{APPENDIX 1}

Proof of Proposition 3.12. For notational ease in the induction proof to follow, it is convenient to make the variable change in (3.6), $y_{n}=x_{1}, \ldots$, $y_{2}=x_{n-1}, y_{1}=x_{n}$. This gives the system

$$
\dot{x}_{1}=x_{2}^{p}, \ldots, \dot{x}_{n-1}=x_{n}^{p}, \dot{x}_{n}=u, \quad Y=\frac{\partial}{\partial x_{n}} .
$$

For $n=1, v\left(x_{1}\right)=(1 / 2) x_{1}^{2}, u^{*}\left(x_{1}\right)=-\left((Y v)\left(x_{1}\right)\right)^{p}=-x_{1}^{p}$, and $h_{p+1}^{+}\left(x_{1}\right)=x_{1}^{p+1}$ suffice.

For $n=k$, assume $v(x)=(1 / 2) x^{T} Q x, Q$ positive definite, and $u^{*}(x)=$ $-\gamma_{0}\left(v_{x_{k}}(x)\right)^{p}$ are such that

$$
\sum_{j=1}^{k-1} x_{j+1}^{p} v_{x_{j}}(x)-\gamma_{0}\left(v_{x_{k}}(x)\right)^{p+1}=-h_{p+1}^{+}(x)
$$

where $h_{p+1}^{+} \in P_{p+1}$ is positive definite, i.e., that $v$ is a Lyapunov function for the system with control $u^{*}$. This will then also be true if $\gamma_{0}$ is replaced by any $\gamma>\gamma_{0}$. Since $v_{x_{k}}$ is a linear function, let $v_{x_{k}}(x)=\sum c_{i} x_{i}$. Note from (ii) that:

$$
\text { If } v_{x_{k}}(x)=0 \text { for some } x \neq 0 \text {, then } \sum_{j=1}^{k-1} x_{j+1}^{p} v_{x_{j}}(x)<0 \text {. }
$$

Next, consider system (i) with $n=k+1$. Make a linear variable change in only the last coordinate, i.e., let $z_{1}=x_{1}, \ldots, z_{k}=x_{k}, c z_{k+1}=x_{k+1}, c>0$ to be chosen. This gives the system

$$
\dot{z}_{1}=z_{2}^{p}, \ldots, \dot{z}_{k-1}=z_{k}^{p}, \dot{z}_{k}=c^{p} z_{k+1}^{p}, \dot{z}_{k+1}=u
$$

where $(1 / c) u$ has been renamed again as $u$. Let

$$
w\left(z_{1}, \ldots, z_{k+1}\right)=v\left(z_{1}, \ldots, z_{k}\right)+(1 / 2)\left(v_{z_{k}}(z)+z_{k+1}\right)^{2} .
$$

Then $w$ is a positive definite, quadratic form, $w_{z_{1}}=v_{z_{1}}+\left(v_{z_{k}}+z_{k+1}\right) c_{1}, \ldots$, $w_{z_{k}}=v_{z_{k}}+\left(v_{z_{k}}+z_{k+1}\right) c_{k}$ and $w_{z_{k+1}}=\left(v_{z_{k}}+z_{k+1}\right)$. We next show that $w$ is a Lyapunov function for (iv) with control $u^{*}(z)=-\gamma\left(w_{z_{k+1}}(z)\right)^{p}, \gamma \geq \gamma_{0}$ to be chosen. Calculating the "trajectory derivative" $\dot{w}$ gives

$$
\begin{aligned}
& \sum_{j=1}^{k-1} z_{j+1}^{p}\left(v_{z_{j}}+\left(v_{z_{k}}+z_{k+1}\right) c_{j}\right) \\
& +c^{p} z_{k+1}^{p}\left(v_{z_{k}}+\left(v_{z_{k}}+z_{k+1}\right) c_{k}\right)-\gamma\left(v_{z_{k}}+z_{k+1}\right)^{p+1} .
\end{aligned}
$$

The expression ( $\mathrm{v}$ ) is homogeneous of degree $(p+1)$ with respect to $\delta_{\varepsilon}^{1}$ hence if it is negative on the unit sphere $S^{k} \subset \mathbb{R}^{k+1}$, it is negative definite and equal to some $-h_{p+1}^{+} \in P_{p+1}, h_{p+1}^{+}$positive definite.

(a) If $z \in S^{k}$ is such that $v_{z_{k}}\left(z_{1}, \ldots, z_{k}\right)=-z_{k+1}=0$, (v) becomes $\sum_{j=1}^{k-1} z_{j+1}^{p} v_{z_{j}}$ which is negative by (iii). 
(b) If $z \in S^{k}$ is such that $v_{z_{k}}=-z_{k+1} \neq 0$, (v) becomes $\sum_{j=1}^{k-1} z_{j+1}^{p} v_{z_{j}}-$ $c^{p} z_{k+1}^{p+1}$. Hence for $c>0$ sufficiently large, expression $(\mathrm{v})$ can be made negative on the compact zero set of $\left(v_{z_{k}}+z_{k+1}\right)$ in $S^{k}$ and therefore (v) is negative on an open neighborhood, $U$, of this set. Then there exists a $\bar{c}>0$ such that $\left(v_{z}+z_{k+1}\right)^{p+1} \geq \bar{c}$ on the compact complement of $U$ in $S^{k}$, and by choosing $\gamma \geq \gamma_{0}$ sufficiently large, the last term on the right of $(\mathrm{v})$ dominates to make the expression (v) negative on $S^{k}-U$.

The required quadratic form for system (i), with $n=k+1$, is then $w\left(x_{1}, \ldots, x_{k},(1 / c) x_{k+1}\right)$; the ASFC is $u^{*}(x)=-\gamma c\left(w_{z_{k+1}}\right)^{p}$ and the induction step is complete.

REMARK 3.21. The proof of the existence of an ASFC for system (20), proposition 3.20 , follows exactly as the proof of proposition 3.20 , above, except for expression (v) which now becomes

$$
\begin{aligned}
& \sum_{j=1}^{k-1}\left(z_{j+1}^{p}+z_{j+1}^{a} z_{j}^{e}\right)\left(v_{z_{j}}+\left(v_{z_{k}}+z_{k+1}\right) c_{j}\right) \\
& +\left(c^{p} z_{k+1}^{p}+c^{a} z_{k+1}^{a} z_{k}^{e}\right)\left(v_{z_{k}}+\left(v_{z_{k}}+z_{k+1}\right) c_{k}\right)-\gamma\left(v_{z_{k}}+z_{k+1}\right)^{p+1} .
\end{aligned}
$$

Now, as in the previous proof, if $z \in S^{k}$ is such that $v_{z_{k}}(z)=-z_{k+1} \neq 0$, from $\left(\mathrm{v}^{\prime}\right)$ one gets

$$
\sum_{j=1}^{k-1}\left(z_{j+1}^{p}+z_{j+1}^{a} z_{j}^{e}\right) v_{z_{j}}(z)-c^{p} z_{k+1}^{p+1}-c^{a} z_{k+1}^{a+1} z_{k}^{e} .
$$

But $(p+1)$ is even, $(a+1)$ and $e$ are even, hence the result follows as in the previous proof.

The author would like to acknowledge the many suggestions, comments and needs for corrections on the first version of this paper, made by an anonymous referee.

\section{RefERENCES}

[1] F. Ancona: Decomposition of homogeneous vector fields of degree one and representation of the flow, Annales de l'Institut Henri Poincaré, J. Nonlinear Analysis (to appear).

[2] M. Bardi: A boundary value problem for the minimum time function, SIAM J. Control and Opt., 27, 1989, 776-785.

[3] R. Bianchini and G. Stephani: Graded Approximations and Controllability along a trajectory, SIAM J. Control and Opt., 28, 1990, 903-924.

[4] R.W. Brockett: Asymptotic stability and feedback stabilization, in Differential Geometric Control Theory, 27, R. Brockett, R.S. Millman, H.J. Sussmann, eds., Birkhäuser, Boston, 181-191, 1983.

[5] J.-M. Coron: A necessary condition for feedback stabilization, Systems and Control Lett., 14, 1990, 227-232.

[6] J.-M. Coron: On the stabilization in finite time of locally controllable systems by means of continuous time-varying feedback laws, SIAM J. Control and Opt., 33, $1995,804-833$.

[7] W. Dayawansa, C. Martin and G. Knowles: Asymptotic stabilization of a class of smooth two dimensional systems, SIAM J. Control and Opt., 28, 1990, 1321-1349. 
[8] W. Dayawansa and C. Martin: Asymptotic stabilization of low dimensional systems, in Systems and Control Theory, C.I. Byrnes and A. Kurzhansky, eds., Birkhäuser, Boston, 53-67, 1991.

[9] L.C. Evans and M.R. James: The Hamilton-Jacobi-Bellman equation for time optimal control, SIAM J. Control and Opt., 27, 1989, 1477-1489.

[10] H. Hermes: Homogeneous coordinates and continuous asymptotically stabilizing feedback controls, in Diff. Eqs., Stability and Control, S. Elaydi, ed., Lecture Notes in Pure and Applied Math., 127, Marcel Dekker Inc., 249-260, 1991.

[11] H. Hermes: Large time local controllability via homogeneous approximation, SIAM J. Control and Opt., 34, 1996, 1291-1299.

[12] H. Hermes: Nilpotent and high-order approximations of vector field systems, SIAM Review, 33, 1991, 238-264.

[13] H. Hermes: Asymptotically stabilizing feedback controls and the nonlinear regulator problem, SIAM J. Control and Opt., 29, 1991, 185-196.

[14] H. Hermes: Asymptotically stabilizing feedback controls, J. Diff. Eqs., 92, 1991, 7689.

[15] M. Kawski: Geometric homogeneity and stabilization, in NOLCOS'95, 1, 164-169, 1995.

[16] J.B. Pomet: Explicit design of time varying stabilizing control laws for a class of controllable systems without drift, Systems 6 Control Letters, 18, 1992, 147-158.

[17] E.B. Lee and L. Markus: Foundations of Optimal Control Theory, John Wiley, N.Y., 1967.

[18] L.P. Rothschild and E.M. Stein: Hypoelliptic differential operators and nilpotent groups, Acta Math., 137, 1976, 247-320.

[19] L. Rosier: Homogeneous Liapunov function for continuous vector field, System and Control Lett., 19, 1992, 467-473.

[20] R. Sepulchre: Contributions to nonlinear control systems analysis by means of the direct method of Liapunov, Ph.D thesis, Universite Catholique de Louvain, 1994.

[21] G. Stefani: Local properties of nonlinear control systems, in Geometric Theory of Nonlinear Control Systems, B. Jakubczyk, W. Respondek, K. Tchon, eds., Tech. Univ., Wroclaw, 219-226, 1984.

[22] H.J. Sussmann: A general theorem on local controllability, SIAM J. Control and Opt., 25, 1987, 158-194.

[23] J. Tsinias: Triangular systems: A global extension of Coron-Praly theorem on the existence of feedback-integrator stabilizers, (preprint) 1996. 